\section{Learning and retention of English words with successive approximations to a complex mnemonic instruction}

\section{P. S. DELIN, University of Adelaide, Adelaide, South Australia}

Groups of Ss learned a list of English words after receiving one of seven different sets of learning instructions and one of two recall instructions constructed in such a way as to represent successive approximations to the instructions given in commercial memory courses. The $S$ s relearned the list after intervals of 5, 10, or 15 weeks. In general, the performance of the Ss was related to the completeness of the instructions they had received. In the learning data, the proportion of errors taking the form of omissions was also related to completeness of instructions.

In a number of commercial memory training courses (e.g., Furst, 1954; Lorayne, 1963) instructions are given for learning serial lists of nouns using imagery mnemonics. In previous studies (e.g., Delin, 1969), it has appeared that $S$ s receiving these instructions perform considerably better than Ss not receiving them and (Delin, 1968), that recall performance is related to the success with which the instructions are carried out.

These instructions are complex, however, in that the $S$ is asked to learn the items pair by pair, to use imagery, to invoke as many sensory modalities as possible, and to make his images vivid, active, and bizarre. Any or most of the elements in these instructions could be irrelevant to the heightened performance of the Ss.

It therefore seemed worthwhile to make an attempt to break down these mnemonic instructions into subinstructions, and to examine the effect of each of these upon performance. The lack of independence of the different instruction elements which could be identified suggested a cumulative rather than a factorial design. Thus you cannot ask a $S$ to make his images bizarre without asking him to make images. Furthermore, the nature of the relations between the different subinstructions is such as to make some orders of cumulation more reasonable than others. Thus, since it is likely that while many vivid images are not bizarre most bizarre images are vivid, the vividness instruction should occur earlier.

On the basis of such considerations as these, seven different sets of learning instructions were prepared, ranging from standard serial anticipation instructions to the complete set of bizarre imagery mnemonic instructions of varying degrees of completeness.

However, it seemed in principle possible for a $S$ to know how to create a mnemonic, but not know how to use it. Therefore, it was decided to run, for each of the six groups that received some mnemonic instructions, a parallel group which also received a recall instruction.

Finally, since some of the subinstructions might have differential effects on learning and retention, it was decided that each $S$ should relearn the list after one of three retention intervals. In order to facilitate the retention aspect of the analysis, it was decided that learning should be for a fixed number of presentations rather than to a preset criterion.

\section{METHOD}

Two 10-item lists of English nouns were used, one for practice (List P), and one experimental list (List E). These lists were low interitem association orderings of items out of two longer lists previously rated for interitem association (see Delin, 1968).

The contents of the learning instructions were as follows: (a) Treat each item as being paired with the previous one. (b) Make a mental image of each item paired with the previous one. (c) Make a vivid mental image of each item with the previous one. (d) Make a vivid, active image of each item with the previous one. (e) Make a vivid, active image of each item with the previous one, using as many sensory modalities as possible. (f) Make a vivid, active, highly bizarre image of each item with the previous one, using as many sensory modalities as possible.

The recall instructions were: (A) In trying to recall, bear in mind the previous item and try to think what you paired it with. (This instruction was only used with Learning Instruction a.) (B) In trying to recall, try to remember what you did with the previous item, and then try to recognize the item you are seeking in the image that springs to mind.

The 13 groups were thus labelled $C, a, b$, $c, d, e, f, a A, b B, c B, d B, e B$, and $f B$, according to their instructions. Each group was further subdivided into three retention groups of seven $\mathrm{Ss}$ who retumed at intervals of 5 weeks, 10 weeks, and 15 weeks.

The Ss were 273 first-year psychology students. Seventeen of these failed to re turn for the retention trials and fresh Ss were run to replace these.

Subjects were run individually. All Ss learned List $P$, which was presented by memory drum at a 5 -sec rate, followed by five anticipation trials at the same rate. Intertrial interval was approximately $10 \mathrm{sec}$. The Ss were placed in one of seven performance categories on the basis of their error scores on this task. They were then allocated randomly to one of the experimental groups, with the restriction that the number of $S s$ in each learning-ability category was to be held constant across groups. They were also assigned randomly, but with the same restriction, to subgroups with one of the three retention intervals.

The Ss were thus divided into 13 groups of 21 . The 13 were made up of a control group (C), with standard serial anticipation instruction, and 12 experimental groups. These latter consisted of six groups having one of the six sets of mnemonic learning instructions (Instructions a-f) and six groups having Instructions a-f plus a recall instruction (A or B).
Table 1

Means and SDs of Error Scores on Leaming (Each $N=21$ ) and Relearning (Each $\mathbf{N}=7$ ), for All 13 Experimental Groups

\begin{tabular}{|c|c|c|c|c|c|c|c|c|}
\hline & \multicolumn{2}{|c|}{ Learning } & \multicolumn{6}{|c|}{ Relearning } \\
\hline & \multirow[b]{2}{*}{ Mean } & \multirow[b]{2}{*}{ SD } & \multicolumn{2}{|c|}{5 week } & \multicolumn{2}{|c|}{10 week } & \multicolumn{2}{|c|}{15 week } \\
\hline & & & Mean & $\mathrm{SD}$ & Mean & $\mathrm{SD}$ & Mean & SD \\
\hline $\mathrm{C}$ & 5.85 & 4.30 & 3.7 & 2.63 & 9.4 & 5.21 & 13.2 & 6.18 \\
\hline a & 5.83 & 4.92 & 4.2 & 5.24 & 11.6 & 4.54 & 12.4 & 4.31 \\
\hline b & 4.19 & 4.49 & 3.8 & 3.18 & 12.7 & 5.61 & 14.7 & 6.05 \\
\hline $\mathrm{c}$ & 3.59 & 3.35 & 4.3 & 5.13 & 9.5 & 4.31 & 8.0 & 3.76 \\
\hline d & 3.21 & 3.40 & 2.5 & 2.72 & 8.0 & 3.55 & 9.4 & 4.13 \\
\hline $\mathrm{e}$ & 2.38 & 2.40 & 1.2 & 3.43 & 6.7 & 2.98 & 7.2 & 3.93 \\
\hline f & 2.57 & 3.57 & 3.1 & 2.47 & 8.2 & 4.35 & 8.1 & 3.71 \\
\hline $\mathrm{aA}$ & 6.04 & 4.00 & 3.8 & 1.96 & 10.3 & 5.19 & 13.1 & 5.21 \\
\hline $\mathrm{bB}$ & 4.00 & 3.79 & 3.0 & 4.11 & 7.9 & 3.44 & 11.4 & 4.43 \\
\hline $\mathrm{cB}$ & 3.33 & 3.33 & 2.2 & 2.10 & 11.6 & 6.71 & 9.8 & 5.10 \\
\hline $\mathrm{dB}$ & 3.52 & 3.34 & 1.9 & 1.04 & 7.3 & 2.93 & 7.4 & 2.87 \\
\hline $\mathrm{eB}$ & 2.76 & 2.33 & 1.4 & 1.22 & 5.1 & 3.67 & 6.8 & 3.33 \\
\hline $\mathrm{fB}$ & 2.52 & 3.09 & 2.9 & 1.28 & 7.2 & 4.77 & 9.3 & 3.56 \\
\hline
\end{tabular}

Note: Learning means are based on three anticipation trials whereas relearning means are based on four anticipation trials. 


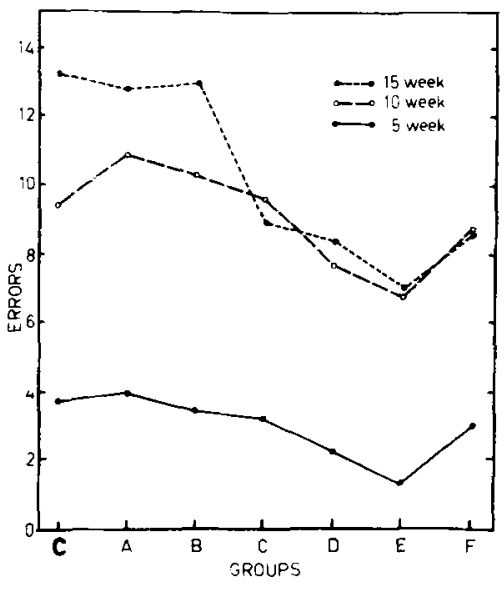

Fig. 1. Mean error scores of retention groups at three intervals with recall instruction and no-recall-instruction groups combined.

Each $S$ was informed that a large number of different sets of instructions were being used in the experiment, and that he should not assume that the instructions he received were expected to facilitate his learning of the experimental list. He was then given the learning instructions, and recall instructions, if any, appropriate to the group to which he had been assigned. List $\mathrm{E}$ was then presented by memory drum at a $7-\mathrm{sec}$ rate, followed by three anticipation trials at the same rate, with an intertrial interval of about $10 \mathrm{sec}$. The $S$ was then cautioned not to rehearse the experimental list and told when he was to retu $m$ for the relearning session.

At the relearning session, each $S$ was given four anticipation trials with the experimental list, under the same conditions as before. He was then briefly interrogated on his application of the instructions he had been given, and on his use of other mediating techniques.

\section{RESULTS}

The main results are summarized in Table 1. For all groups, the error scores were strongly skewed, and they were transformed for the purposes of further analysis by adding 1.0 , to eliminate zeroes, and then taking logs.

A two-way analysis of variance was carried out on the error scores of the 12 experimental groups (i.e., the six learning instructions against the two recall instructions). The learning-instructions effect proved highly significant $(F=5.17$, $\mathrm{df}=5 / 240, \quad \mathrm{p}<.001)$. The recall instructions had negligible effect $(F=.055$, $\mathrm{df}=1 / 240$ ), and the groups with and without recall instructions were pooled for further analysis. A one-way analysis of variance using these six pooled-instruction groups plus the control group proved significant $(F=6.01, \mathrm{df}=6 / 266, \mathrm{p}<.001)$. Post hoc comparisons were carried out using the method of Scheffé (Hays, 1963). Comparing individual pairs, the only significant differences $(p<.05)$ were those between Group $C$ and Groups $\mathrm{e}+\mathrm{eB}$ and $f+f B$, and between Group $a+a A$ and Groups $e+e B$ and $f+f B$. Further Scheffé tests were carried out comparing all groups above and below each point on the dimension of completeness of mnemonic instructions. All of these comparisons were significant except those between Group C and the rest, and between Group $f+f B$ and the rest. To summarize these analyses, they suggest that the recall instructions did not affect performance, and that each addition to the learning instructions, with the exception of the bizarreness instruction, and the possible exception of the pairing instruction, produced an improvement in learning performance.

It was found in a previous study (Delin, 1969) that Ss with mnemonic instructions made a higher proportion of their errors in the form of omissions than did control Ss. It seemed worthwhile to see whether this tendency increased with the completeness of the mnemonic instructions. The natural way to look at this would have been to see whether there was a trend between groups in the scores obtained by expressing omission errors as a proportion of total errors. However, a number of Ss had made either no errors at all, or no errors of omission, so the much weaker alternative was used of asking whether the proportion of Ss making inore errors of omission than of commission increased as the mnemonic instructions became more complete. This hypothesis was tested by means of the rank t test (Bross, 1954) and proved significant at the .005 level $\left(t^{2}=9.40\right)$, strongly suggesting a trend in the expected direction. When this procedure was carried out separately on the data for the first anticipation trial and for later anticipation trials both proved significant $\left(\mathrm{t}^{2}=5.41, \quad \mathrm{p}<.02\right.$, and $\left.\mathrm{t}^{2}=7.22, \mathrm{p}<.01\right)$. Thus, it would be difficult to account for the observed trend in terms of an interaction between a tendency for all Ss to make omission errors in the early trials, and a tendency for mnemonic Ss to make less errors than control Ss in later trials.

The relearning data are summarized in Table 1. As with the learning data, the scores were strongly skewed, and further analysis was carried out on the raw scores transformed by adding 1 , and taking logs. A three-way analysis of variance (three retention intervals, recall instruction vs no-recall instruction, and six levels of learning instruction) found significant effects for learning instruction $(F=4.92$, $\mathrm{df}=5 / 216, p<.001)$ and for retention interval $(F=5.47, \mathrm{~d} f=2 / 216, \mathrm{p}<.001)$. The interaction between these two factors nearly reached significance at the .05 level $(F=1.85, \mathrm{df}=10 / 216$, critical $F=1.88)$. The groups with and without recall instructions were again combined, and the means of the resultant groups, and the control groups, form the basis of Fig. 1. The convergence of the lines for the 15 -week and 10-week groups evidently corresponds to the near-significant interaction reported above. Trend tests (Jonckheere, 1953) were carried out separately for the three retention intervals, and proved significant at the .05 level for the 15-week and 10-week intervals, and just below significance $\left(\mathrm{S}_{\mathrm{o}}=1.95\right.$, critical $S_{o}=1.96$ ) for the 5-week interval. The drop in performance associated with the $\mathrm{f}$ (bizarreness) instruction is unlikely to have been statistically artifactual, since it occurred independently for all six f-instruction subgroups.

An analysis of the errors of omission and commission made during relearning disclosed no systematic between-groups differences. Overall relative proportions of errors which were errors of commission were .291 for the first anticipation trial, and .592 for later trials. The equivalent proportions for the learning data were .338 and .541 .

Subjective reports collected from the Ss suggested that most of the control Ss had used some form of natural language mediator to learn at least some of the items, but that very few of the experimental Ss had used techniques other than the one suggested in the instructions. Many of the experimental Ss reported that they had experienced difficulty in carrying out the instructions, and this was especially true of the $S$ receiving the finstruction. Of these 42 Ss, 7 said that they had been unable to make any images, and only 10 were able to describe images which the $E$ would have classified as being in any way bizarre.

\section{DISCUSSION}

In spite of the use of low interitem association lists, much of the detailed analysis that should have been possible with this experimental design was vitiated by the relative paucity of errors. On the other hand, from the point of view of the f-instruction Ss, the task appears to have been difficult, as they seem not to have been able to carry out the instructions. This observation suggests some caution in the interpretation of studies (e.g., those of Wood, 1966) in which mediation instructions were given, but for which no information is available about the success of Ss in carrying out the instructions under the given conditions. Thus, the results of the present study, in the absence of this qualification, would suggest that bizarreness, if it has any effect, reduces the effectiveness of imagery mnenomics. It is worth noting in this connection that in a 
previous study (Delin, 1968) it was found that ratings of descriptions of images for the degree to which they conformed to bizarre-image mnemonic instructions related positively to recall performance.

Apart from the anomalous performance of the f-instruction Ss, the results of the present study suggest that as the mnemonic instructions become more complete, the differences between control and experimental Ss become greater in terms both of level of performance and of relative preponderance of omission and commission errors. The differences in error type did not appear in the relearning data, but this fact is not easy to interpret, since these data confound recall and relearning per se. Not enough errors were made in relearning for it to be possible to analyze these factors separately. However, even if the mnemonic/control difference in predominant error type only occurs on learning, it suggests that mnemonic learning is different from rote learning in kind as well as in degree-an observation which is interesting in view of the fact that Ss frequently, and spontaneously, report that mnemonic learning "feels" quite different from "ordinary" learning.

\section{REFERENCES}

BROSS, I. D. J. Is there an increased risk? Federation Proceedings, 1954, 13, 81 5-819.

DELIN, P. S. Success in recall as a function of success in implementation of mnemonic instructions. Psychonomic Science, 1968, 12, 153-154.

DELIN, P. S. The learning to criterion of a serial list with and without mnemonic instructions. Psychonomic Science, 1969, 16, 4, 169-170.

FURST, B. Stop forgetting. New York: Garden City Press, 1954.

HAYS, W. L. Statistics for psychologists. New York: Holt, Rinehart \& Winston, 1963.

JONCKHEERE, A. R. A distribution-free $\mathrm{k}$-sample test against ordered alternatives. Biometrika, 1953, 40, 133-145.

LORAYNE, H. Develop a super-power memory. London: The New English Library, 1963.

WOOD, G. Mnemonic systems in recall. Unpublished doctoral dissertation, Northwestern University, 1966.

Vermont were tested in one of three conditions, $16 \mathrm{Ss}$ each. There were three decks of 32 index cards, each deck having all combinations of five two-valued dimensions, so that each card bore five words, one from each dimension. The dimensions and values are given in Table 1.

Conditions were ID (intradimensional shift), ED (extradimensional shift), and control (shift to all new dimensions). For the preshift task, ID and ED groups had the experimental deck and controls the control deck. Then all groups had the postshift deck. Both decks for ID and ED conditions had the same dimensions. The ID group shifted to new values on the same dimension as before, while the ED group shifted to new values on another of these dimensions. Controls shifted to an entirely new set of dimensions. Each $\mathrm{S}$ was assigned a condition and solution in the order of his appearance. Cards were shown singly and $S$ was to name the correct word on each, and $E$ indicated whether the response was right. After 10 consecutive correct responses $S$ was switched to the postshift deck and taken to the same criterion.

\section{RESULTS}

Preshift mean trials up to the criterial run were $4.3,4.5$, and 3.9 for ID, ED, and controls, respectively. A nonsignificant $F$ indicated comparable performances. Postshift mean trials were $0.0,2.8$, and 1.7 for ID, ED, and controls. Since the ID group had zero variance, the Kruskal-Wallis nonparametric ANOVA was used and showed $\mathrm{H}(2)=27.0, \mathrm{p}<.001$.

\section{An Improved discrimination-shift design'}

\author{
NORMAN J. SLAMECKA, State University \\ of New York, Buffalo, N.Y. 14214
}

$A$ discrimination-shift experiment was performed whose design was free of several sources of bias. All postshift values differed from preshift values, and the stimuli were words. Three groups were used: the $I D$, shifted to the same dimension; the ED, shifted to another dimension; and the control, shifted to entirely new dimensions. Results showed positive transfer for $I D$ and negative for $E D$, strongly supporting a mediational interpretation.
A recent review of shift paradigms testing single-stage vs mediational interpre tations of human discrimination learning, stressed several potential biases (Slamecka, 1968). These involved intermittent reinforcement, shift detectability, obviousness of solution, stimulus novelty, and negative transfer. An improved design was suggested, where all postshift dimensions have values different from the preshift task, and the stimuli are words. The present experiment demonstrates such a design.

\section{METHOD}

Forty-eight students at the University of

Table 1

Makeup of Decks. Each Card had a Word from Each Dimension.

\begin{tabular}{|c|c|c|c|c|c|}
\hline \multicolumn{4}{|c|}{ Preshift } & \multicolumn{2}{|c|}{ Postshift } \\
\hline \multicolumn{2}{|c|}{ Control } & \multicolumn{2}{|c|}{ Experimental } & & \\
\hline football & tennis & monkey & lion & racoon & deer \\
\hline sulphur & chlorine & radish & carrot & potato & lettuce \\
\hline purple & white & Chicago & Atlanta & Baltimore & Omaha \\
\hline triangle & hexagon & trumpet & violin & piano & guitar \\
\hline Chevrolet & Pontiac & William & Henry & Edward & Matthew \\
\hline
\end{tabular}

\section{DISCUSSION}

Since the ID group showed positive transfer and the ED group gave negative transfer, this supports a mediational interpretation. However, it is conceivable that the results came about by direct free associations between the preshift and postshift words, tending to facilitate ID and hinder ED performance. To check this, 100 new Ss were asked to give free associates to all words from both preshift decks. Out of the 2,000 responses a total of only nine $(0.4 \%)$ were postshift words, whereas $20 \%$ were appropriate dimensional terms. Thus, the direct association possibility in unlikely. It is concluded that the data, coming from a design free of the aforementioned biases, strongly support a mediational interpretation.

\section{REFERENCE}

SLAMECKA, N. J. A methodological analysis of shift paradigms in human discrimination learning. Psychological Bulletin, 1968, 69, 423-438.

\section{NOTE}

1. Miss Jean Stables collected the data. The paper was written during the author's tenure as a NIH Special Fellow at the Institute of Human Learning, Berkeley. 